\title{
Anticipatory Guidance for Children with Haemophilia
}

\author{
Nirmala Venkata*, Saikrishna Degala and Nuvvula Sivakumar
}

JSS Dental College and Hospital, Mysore, Karnataka, India

\begin{abstract}
Anticipatory guidance as a proactive developmentally based counselling technique that focuses on the need of a child at each stage of life. The prevention of dental problems is essential component of oral care particularly for haemophilic children. Parents should be educated and motivated regarding the value of prevention of dental caries, gingival and periodontal diseases, as well as dental trauma. Teething toys like a firm rubber or water filled plastic teething rings used during teething. Brushing twice daily with a fluoride toothpaste.1,000-ppm fluoride toothpaste for children under 7 years of age and 1,400-ppm fluoride toothpaste for people over 7 years of age. High sugar or acid content should be limited to mealtimes Most of the restorative procedures done without factor replacement. Use thin rubber dam, avoid retainers with sub gingival extensions, to protect interdental papilla wedged can be used. High speed vacuum saliva ejector should be used cautiously. Pulpotomy and pulpectomy is preferable to extraction. However, 30$40 \%$ factor replacement is administered one hour prior to the extraction. This article discuss about anticipatory guidance in children with haemophilia.
\end{abstract}

Keywords: Anticipatory guidance; Children; Dental treatment; Haemophilia

\section{Introduction}

Nowak describes anticipatory guidance as a proactive, developmentally based counselling technique that focuses on the need of a child at each stage of life [1].

When it effectively means is that one should not get pessimistic for many times a patient may lack cooperation at this young age. Providing an insight into the development of a child will involve the parent, with a much more focused strategy. Also, at every stage it is essential that the dentist takes into consideration the various milestones of dental development. Such anticipatory guidance can make the parents more at ease during childhood dental visit; these pointers are also essentials in preventing many of the possible dental problems children would otherwise often face.

Parents should be educated and motivated regarding the value of prevention of dental caries, gingival and periodontal diseases, as well as dental trauma. Caries prevention plan should be developed separately for each child with bleeding disorders. American academy of paediatric dentistry developed a caries risk assessment tool which is a very important asset for these children.

Teething toys like a firm rubber or water filled plastic teething rings are available commercially which have been used during teething. These rings will help the child erupt their teeth through gingiva. Children with bleeding disorders freezed teething rings should be used as it may improve vasoconstriction and also helps gingiva from oozing [2].

Parents should be supervised during brushing of their children; soft nylon bristled tooth brush and circular motion is advisable, they should brush gently not a sawing motion which may damage or cut the gingiva. Use of an electric tooth brush is very important in Haemophilic children with hemarthosis of the elbow joint may not be able to use manual tooth brush. Rinsing with cold water usually stop the occasional gingival bleed. Parents as well as their children should be aware that the child should not brush their gums. Care should be taken especially not brush their gums in the areas where there is bleeding from the gums. Brushing technique should be demonstrated so that adequate gingival stimulation occurs while brushing. Gums that are not brushed become swollen, inflamed, and puffy and bleed very easily [3]. In order to prevent inflammation of the gums, it needs to be massaged gently using

The key to manage oral health of children with bleeding disorders is prevention. Identification and early diagnosis of incipient caries should be managed by fluoride application, carious lesions progress to the stage where restorations are indicated, preventive resin restorations, a traumatic restorative treatment, remineralisation and arresting the caries should be follows.

\section{Prevention}

The prevention of dental problems is essential component of oral care. A successful regimen will reduce the need for treatment and should reduce the number of emergency visits [4]. Dental prevention depends on a number of different $\mathrm{F}$ factors some of these may know not be available in developing countries but are included to demonstrate the ideal situation. Brushing twice daily with a fluoride toothpaste 1,000-ppm fluoride toothpaste for children under 7 years of age and 1,400-ppm fluoride toothpaste for people over 7 years of age. The use of fluoride toothpaste depends on the fluoride concentration in the water supply as well as the use of additional fluoride supplements. It should not be use difluoride supplements are taken or if the water supply has a fluoride content of $1 \mathrm{ppm}$ or more. Fluoride supplements may be used, but are not recommended if the water supply has a fluoride content of $1 \mathrm{ppm}$ or more. Supplements include: Fluoride drops, Fluoride tablets Topical application of fluoride using trays Fluoride mouth rinses which can be used on either a daily or a weekly basis. The consumption of foods and drinks with a high sugar or acid content should be limited to mealtime 5 three exposures per day is the recommended maximum. The aim is to ensure that the intake of food and drink does not cause the $\mathrm{pH}$ of the oral cavity to fall below the critical level of $\mathrm{pH} 5$ [5]. The toothbrush should have medium texture bristles because hard bristles

*Corresponding author: Nirmala Venkata, Professor of JSS Dental College and Hospital, Mysore, Karnataka, India, Tel: 91940206976; E-mail: drnirmalavenkata@gmail.com

Received August 07, 2017; Accepted April 13, 2018; Published April 16, 2018

Citation: Venkata N, Degala S, Sivakumar N (2018) Anticipatory Guidance for Children with Haemophilia. J Health Med Informat 9: 309. doi: 10.4172/2157-7420.1000309

Copyright: ( 2018 Venkata N, et al. This is an open-access article distributed under the terms of the Creative Commons Attribution License, which permits unrestricted use, distribution, and reproduction in any medium, provided the original author and source are credited. 
can cause abrasion of the teeth and soft bristles are inadequate to remove plaque. Interdental cleaning aids, such as floss, tape, and interdental brushes, should be used to prevent the formation of dental caries and periodontal disease $[6,7]$.

\section{Dental treatment}

It is essential to prevent accidental damage to the oral mucosa when carrying out any procedure in the mouth. Injury can be avoided by: Careful use of saliva ejectors;

Careful removal of impressions; Care in the placement of X-ray films, particularly in the sublingual region; Protection of soft tissues during restorative treatment by using a rubber dam or applying yellow soft paraffin $\left(\right.$ Vaseline $\left.^{\circledast}\right)[8]$.

\section{Periodontal treatment}

Healthy periodontal tissue is essential to prevent bleeding and tooth loss. If oral hygiene is poor treatment must start as soon as possible after the patient has had a dental examination and treatment plan formulated to prevent additional damage to the periodontal tissues. In cases of severe periodontal disease, it may be necessary to carry out supragingival scaling initially along with oral hygiene education [9]. Sub gingival scaling can start as soon as the inflammation has decreased. The treatment may need to be carried out over several visits to prevent excessive blood loss. In addition, chlorhexidine gluconate mouthwash can be used to control periodontal problems. Antibiotics may be required to help reduce the initial inflammation. Blood loss of all kinds can be controlled locally with direct pressure or periodontal dressings with or without topical antifibrinolytic agents. Periodontal surgery in patients with bleeding disorders must always be regarded as a highrisk procedure with a significant risk of blood loss. It should only be considered were conservative treatment has failed and oral hygiene is good. Periodontal surgery can be a greater challenge to haemostasis than a simple extraction. The procedure must be carefully planned and the risk is fully explained to the patient $[10,11]$.

\section{Removable prosthodontics}

Patients with bleeding disorders can be given dentures as long as they are comfortable. If a partial denture is provided it is important that the periodontal health of the remaining teeth is maintained.

\section{Orthodontic treatment}

Fixed and removable orthodontic appliances may be used along with regular preventive advice and hygiene therapy. Special care should be taken when treating patients with a severe bleeding disorder to ensure that the gingiva is not damaged when fitting the appliance. Avoid of protruding sharp edges and wires.

\section{Restorative procedures}

Restorative treatment can be undertaken routinely providing care is taken to protect the mucosa. There is a risk of bleeding with the use of matrix bands or wooden wedges. This can be controlled by local means or the application of topical agents. Most of the restorative procedures done without factor replacement. Use thin rubber dam; avoid retainers with sub gingival extensions, to protect interdental papilla wedges can be used, while using matrices care should be taken in order to prevent bleeding. High speed vacuum saliva ejector should be used cautiously. Use wet cotton, while taking intra oral periapical radio graphs not to cause harm to sublingual tissues. Prefer apex locators for working length. Retraction cord should be used during crown preparation; wax should be used on the periphery of the impression tray [12].

\section{Endodontics}

Endodontic treatment is generally low risk for patients with bleeding disorders. If a pulpectomy is indicated, the possibility of the tooth requiring conventional endodontic treatment must also be considered it is important that the procedure should be carried out carefully with the working length of the root canal calculated to ensure that the instruments do not pass through the apex of the root canal. The presence of bleeding in the canal is indicative of pulp tissue remaining in the canal. Sodium hypochlorite should be used for irrigation in all cases, followed by the use of calcium hydroxide paste to control the bleeding. Formaldehyde derived substances may also be used in cases where there is persistent, bleeding or even before the pulpectomy [13].

Pulpotomy and pulpectomy is preferable than extraction. These procedures can be successfully completed using local infiltration intrapulpal injection used safely to control pain. Intracanal bleeding is controlled by calcium hydroxide placement and if pulp tissue is necrotic local anesthesia is not required [14].

\section{Anesthesia and pain management}

Dental pain can usually be controlled with a minor analgesic such as paracetamol (acetaminophen). Aspirin should not be used due to its inhibitory effect on platelet aggregation. The use of any non-steroidal anti-inflammatory drug (NSAID) must be discussed beforehand with the patient's haematologist because of their effect on platelet aggregation. There are no restrictions regarding the type of local anaesthetic agent used although those with vasoconstrictors may provide additional local haemostasis. It is important to advise patients and parents of children about the risks of local oral trauma before the anaesthetic wears off. A buccal infiltration can be used without any factor replacement. It will anesthetize all the upper teeth and lower anterior and premolar teeth [15].

The mandibular molar teeth are usually treated using the inferior alveolar nerve block. This should be given only after raising clotting factor levels by appropriate replacement therapy, as there is a risk of bleeding into the muscles along with potential airway compromise due to a hematoma in the retro molar or pterygoid space. The intra ligamental technique or interosseous technique should be considered instead of the mandibular block. Articaine has been used as a buccal infiltration to anesthetize the lower teeth. A lingual infiltration also requires appropriate factor replacement since the injection is into an area with a rich plexus of blood vessels and the needle is not adjacent to bone. There is a risk of a significant airway obstruction in the event a bleed. No haemostatic cover is required for buccal infiltration, intrapapillary and intraligamentary injections and other procedures like inferior nerve block and lingual infiltration haemostatic cover is not required. Surgical treatment, including a simple dental extraction, must be planned to minimize the risk of bleeding, excessive bruising, or hematoma formation. The following points will help prevent problems: Emergency surgical intervention in dentistry is rarely required as pain can often be controlled without resorting to an unplanned treatment. All treatment plans must be discussed with the haemophilia unit if they involve the use of prophylactic cover [16-20].

\section{Conclusion}

The paediatric dentist needs to provide the full range of dental treatment and care for children with bleeding disorders and he should do this in conjunction with paediatric haematologist to discuss about number of dental procedures in the treatment plan that may cause bleeding, local anaesthetic administration and number of appointments needed to complete treatment. Hence the quality of life will be improved. 
Citation: Venkata N, Degala S, Sivakumar N (2018) Anticipatory Guidance for Children with Haemophilia. J Health Med Informat 9: 309. doi: 10.4172/2157-7420.1000309

Page 3 of 3

\section{References}

1. Nowak AJ, Cassamassimo PS (1995) Using Anticipatory Guidance to provide early dental intervention. J Am Dent Assoc 126: 1156-1163.

2. Pinkham, Cassamassimo, Fields, McTigue, Nowak. Pediatric Dentistry Infancy through Adolescence. 4th edition, W.B. Saunders Publications.

3. Jeffery A, McDonald RE, Avery DR (2010) McDonald and Avery Dentistry for the Child and Adolescent 9th Edition. Elsevier 3: 1-5.

4. Brewer AW, Roebuck EM, Donachie M (2003) The dental management of children with haemophilia and other congenital bleeding disorders. Haemophilia's 96: 73-77.

5. Evas BE, Aledort LM (1978) Hemophilia and dental treatment. J Am Dent Assoc 96: 827-834

6. Soucie JM, Evatt B, Jackson D (1998) Occurrence of Hemophilia in the United States. The Hemophilia Surveillance System Project Investigaters. Am J Hematol 59: 288-294.

7. Miller R (1999) Counselling about diagnosis and inheritance of genetic bleeding disorders: haemophilia A and B. Haemophilia 5: 77-83.

8. Sonis AL, Musselman RJ (1982) Oral bleeding in classic hemophilia. Oral Surg Oral Med Oral Pathol 53: 363-366.

9. Kessler CM (2008) Advances in the treatment of hemophilia. Adv Hemato Oncol 6: 184-187.

10. Young G, Aledort L (2005) Therapy for haemophilia: recent advances and goals for the future. Expert Opin Emerg Drugs 10: 173-184.
11. Street AM, Ljung R, Lavery SA (2008) Management of carriers and babies with Haemophilia. Haemophilia 14: 181-187.

12. Kumar JN, Kumar RA, Varadarajan R, Sharma N (2007) Specialty dentistry for the haemophiliac: is there a protocol in place? Indian J Dent Res 18: 48-54.

13. Dudeja PG, Dudeja KK, Lakhanpal M, Ali S (2014) Endodontic management of a Haemophilic patient-A clinical perspective. JCDR 8: ZD17.

14. Shastry SP, Kaul R, Baroudi K, Umar D (2016) Haemophilia A: Dental consideration and management. Jol of Inti Sco Prev Commu Dent 34: 82-86.

15. Lee AP, Boyle CA, Savidge GF, Fiske J (2005) Effectiveness in controlling haemorrhage after dental scaling in people with haemophilia by using tranexamic acid mouthwash. Br Dent J 98: 33-38.

16. Israels S, Schwetz N, Boyar R, McNicol A (2006) Bleeding disorders: characterization, dental considerations and management. J Can Dent Assoc 9: 827-829.

17. Azhar S. Periodontal status and IOTN interventions among young haemophiliacs. Haemophilia 5: 55-59.

18. Correa ME, Annicchino-Bizzacchi JM, Jorge J Jr, Paes de Almeida O, Ozelo MC (2006) Clinical impact of oral health indexes in dental extraction of haemophilic patients. Oral Maxillofac Surg 64: 785-788.

19. Brewer AK (2008) Advances in minor oral surgery in patients with congenital bleeding disorders. JADA 9: 119-121.

20. Piot B, Sigaud-Fiks M, Huet $P$, Fressinaud E, Trossaërt M, et al. (2002) Management of dental extractions in patients with bleeding disorders. Oral Surg Oral Med Onal Pathol Oral Radiol Endod 93: 247-250. 